\title{
Identification of differentially expressed genes affecting hair and cashmere growth in the Laiwu black goat by microarray
}

\author{
JINSHAN ZHAO ${ }^{1-3^{*}}$, HEGANG LI ${ }^{1,2^{*}}$, KAIDONG LIU ${ }^{2}$, BAOXUN ZHANG ${ }^{2}$, PEIPEI LI ${ }^{2}$, \\ JIANNING HE ${ }^{1}$, MING CHENG ${ }^{2}$, WEI DE ${ }^{4}$, JIFENG LIU ${ }^{1}$, YAOFENG ZHAO ${ }^{3}$, LIHUA YANG ${ }^{5}$ and NAN LIU ${ }^{1}$ \\ ${ }^{1}$ College of Animal Science and Technology, Qingdao Agricultural University, Qingdao, Shandong 266109; \\ ${ }^{2}$ Department of Reproduction and Breeding, Qingdao Institute of Animal Science and Veterinary Medicine, \\ Qingdao, Shandong 266100; ${ }^{3}$ College of Biology, China Agricultural University, Beijing 100193; \\ ${ }^{4}$ College of Basic Medicine, Nanjing Medical University, Nanjing, Jiangsu 210002; ${ }^{5}$ College of Biotechnology, \\ Inner Mongolia Agricultural University, Hohhot, Nei Mongol Autonomous Region 010018, P.R. China
}

Received September 8, 2015; Accepted June 30, 2016

DOI: $10.3892 / \mathrm{mmr} .2016 .5728$

\begin{abstract}
Goats are an important source of fibers. In the present study microarray technology was used to investigate the potential genes primarily involved in hair and cashmere growth in the Laiwu black goat. A total of 655 genes differentially expressed in body (hair-growing) and groin (hairless) skin were identified, and their potential association with hair and cashmere growth was analyzed. The majority of genes associated with hair growth regulation could be assigned to intracellular, intracellular organelle, membrane-bound vesicle, cytoplasmic vesicle, pattern binding, heparin binding, polysaccharide binding, glycosaminoglycan binding and cytoplasmic membrane-bound vesicle categories. Numerous genes upregulated in body compared with groin skin contained common motifs for nuclear factor 1A, Yi, E2 factor (E2F) and cyclic adenosine monophosphate response element binding (CREB)/CREB $\beta$ binding sites in their promoter region. The promoter region of certain genes downregulated in body compared with groin skin contained three common regions with LF-A1, Yi, E2F, Collier/Olfactory-1/early B-cell factor 1, peroxisome proliferator-activated receptor $\alpha$ or $\mathrm{U}$ sites. Thus, the present study identified molecules in the
\end{abstract}

Correspondence to: Mrs. Lihua Yang, College of Biotechnology, Inner Mongolia Agricultural University, 306 Zhaowuda Road, Saihan, Hohhot, Nei Mongol Autonomous Region 010018, P.R. China

E-mail: yanglihua1971@126.com

Dr Nan Liu, College of Animal Science and Technology, Qingdao Agricultural University, 700 Changcheng Road, Chengyang, Qingdao, Shandong 266109, P.R. China

E-mail:nanliu@sina.com

${ }^{*}$ Contributed equally

Key words: hair and cashmere growth, goat, gene expression, cDNA microarray, common motif cashmere-bearing skin area of the Laiwu black goat, which may contribute to hair and cashmere traits.

\section{Introduction}

The domestic goat (Capra hircus) is reared throughout the world, particularly in China, India and other developing countries (1), and serves as an important source of fibers and pelts (2). The Laiwu black goat, of which there are 230,000, is indigenous to Shandong and supplies cashmere, pelts and meat. The advantages of this breed include the high-quality cashmere produced, resistance to disease and a high reproductive rate. With regards to cashmere, the fleece fiber diameter is $<12 \mu \mathrm{m}$, cashmere length is up to $56 \mathrm{~mm}$, and the maximum cashmere yield is $300 \mathrm{~g} \mathrm{(3).} \mathrm{The} \mathrm{cashmere}$ growth in Laiwu black goats begins in late July, continuing to March (3).

The identification of genes involved in cashmere production may provide an opportunity to improve production efficiency and product quality and diversity. This may be accomplished through breeding programs, the development of transgenic lines or via the manufacture of therapeutic agents that improve fibers by altering gene expression (4). Previous studies have investigated the effect of genetic polymorphisms (single nucleotide polymorphisms and quantitative trait loci) on cashmere growth and regulation $(5,6)$. Goats have two types of hair follicles: The primary hair follicle, which produces coarse coat hair and the secondary hair follicle, which produces cashmere (1). Comparative transcriptomic analysis identified 51 genes that were differentially expressed (DE) in primary and secondary hair follicles (1). In addition, microRNAs and protein coding genes with potential roles in goat and sheep hair growth have been identified (7-13). The present study used the Agilent sheep gene expression array to identify genes and proteins associated with wool follicle growth and cycling (14,15). The Agilent sheep gene expression array has previously been performed on goat samples to investigate the contribution of mammary epithelial cells to the immune response during the early stages of Staphylococcus aureus infection (16). 
To date, to the best of our knowledge, no microarray or other transcriptomic analysis has been conducted to investigate hair and cashmere growth in various skin areas of adult goats. In addition, only a limited number of studies have described the molecular characteristics of cashmere growth in the Laiwu black goat $(11,12,17)$. The aim of the present study was to analyze and compare gene expression levels in body (hair and cashmere rich) and groin skin (no hair or cashmere) during the cashmere growth period using microarray technology. This may enable the identification of genes potentially associated with hair and cashmere growth regulation in the Laiwu black goat.

\section{Materials and methods}

Sampling. The protocol of the present study was approved by the Ethics Committee of Qingdao Agricultural University (Qingdao, China). The goats were obtained from Qingdao Aote Sheep Farm (Qingdao, China), and housed in semi-closed sheep houses. Sampling was performed as described previously (14). Sampling was performed in August, during the cashmere growth period, on one ram and two ewes, aged 2 years. Full thickness skin from the body (cashmere/wool-bearing) and groin (no cashmere/wool) were sampled from the three animals under local anesthesia ( $2 \mathrm{ml} 0.5 \%$ procaine hydrochloride; Harbin Pharmaceutical Group Co., Ltd., Harbin, China) for complementary DNA microarray experiments.

RNA preparation, microarray hybridization and data analysis. Total RNA was isolated from the skin samples using TRIzol ${ }^{\circledR}$ reagent (Invitrogen; Thermo Fisher Scientific, Inc., Waltham, MA, USA) according to the manufacturer's instructions. RNA integrity was confirmed by denaturing agarose gel electrophoresis and RNA was quantified using a NanoDrop ${ }^{\circledR}$ ND-1000 Spectrophotometer (NanoDrop Technologies; Thermo Fisher Scientific, Inc.). Total RNA $(2.5 \mu \mathrm{g})$ from each sample was doubled and transcribed into fluorescent complementary RNA using a Quick Amp Labeling kit (Agilent Technologies, Inc., Santa Clara, CA, USA) according to the manufacturer's instructions. Subsequently, RNA samples were incubated with the Agilent Sheep Gene Expression Microarray (Agilent Technologies, Inc.). The microarray signals were scanned and analyzed as previously described (14). Clustering analysis of DE genes was performed using Cluster software version 3.0 (bonsai.hgc.jp/ mdehoon/software/cluster/software. htm) $(18,19)$ to analyze the similarity in the expression patterns among different skin areas. The functional annotation of DE genes was performed using the Database for Annotation, Visualization and Integrated Discovery (DAVID) gene annotation tool version 6.6 (david.abcc.ncifcrf.gov/) (20).

Reverse transcription-quantitative polymerase chain reaction $(R T-q P C R)$ verification. Equal amounts of RNA were reverse-transcribed using Superscript III (Invitrogen; Thermo Fisher Scientific, Inc.). qPCR was performed with SYBR Green Master Mix (Roche Applied Science, Penzberg, Germany) and gene-specific primers. The cycling conditions consisted of an initial, single cycle for $5 \mathrm{~min}$ at $95^{\circ} \mathrm{C}$, followed by 40 cycles of $20 \mathrm{sec}$ at $95^{\circ} \mathrm{C}, 30 \mathrm{sec}$ at $60^{\circ} \mathrm{C}$ and $30 \mathrm{sec}$ at $72^{\circ} \mathrm{C}$. The primer sequences, melting temperatures and product sizes are listed in Table I. GAPDH served as the housekeeping gene, and results were normalized using the $\Delta \Delta \mathrm{Cq}$ method (21).

Network analysis. Groups of genes were analyzed using the Meme Software Suite version 4.10 .2 (meme-suite.org/) to identify common motifs and cis-regulatory elements (22). Common motifs and cis-regulatory elements were searched by Patch in the TRANSFAC ${ }^{\circledR}$ Public version 7.0 database (gene-regulation.com/pub/databases.html) to identify transcription factor binding sites (23).

Statistical analysis. Comparisons between groups were performed using Student's $t$-tests in Microsoft Excel 2007 (Microsoft Corporation, Redmond, WA, USA). Data are expressed as the mean \pm standard deviation. $\mathrm{P} \leq 0.05$ was considered to indicate a statistically significant difference.

\section{Results}

Comparative transcriptome analysis between body and groin skin. Skin samples collected from the two skin areas were analyzed. A total of 15,008 transcripts $(98.68 \%$ of all probe sets) were detected in the two skin areas (data not shown). Following normalization and statistical analyses, 655 genes were identified as DE, with 217 upregulated and 438 downregulated in body compared with groin skin, over the threshold of fold-change ( $>2.0$; data not shown).

To further investigate the similarity in expression patterns of gene transcripts in the two skin areas, cluster analysis was performed using the Cluster 3.0 tool. As presented in Fig. 1, cluster analysis revealed differences between body and groin skin.

Using DAVID, 22 of the DE genes were classified into 16 categories according to their functional correlation (data not shown). A number of these genes could be classified into more than one category. The majority of the genes associated with hair growth regulation could be assigned into the intracellular, intracellular organelle, membrane-bound vesicle, cytoplasmic vesicle, pattern binding, heparin binding, polysaccharide binding, glycosaminoglycan binding and cytoplasmic membrane-bound vesicle categories. Other DE genes, not classified in DAVID, are discussed below.

Confirmation of DE genes by RT-qPCR. To confirm the microarray data, six DE genes were selected for assessment of their relative expression levels by RT-qPCR: Antrum muscle protein 18 (gastrokine 1; GKN1; Fig. 2A), cytochrome P450 family 1 subfamily A member 1 (CYP1A1; Fig. 2B), C-jun (Fig. 2C), Connexin 43 (Fig. 2D), integrin subunit $\beta 1$ (ITGB1; Fig. 2E) and complement component 3 (C3; Fig. 2F). GKN1, CYP1A1 and C-jun were significantly upregulated in body compared with groin skin $(\mathrm{P}=0.013, \mathrm{P}=0.025$ and $\mathrm{P}=0.005$, respectively). Connexin 43, ITGB1 and C3 were significantly downregulated in body compared with groin skin $(\mathrm{P}=0.041$, $\mathrm{P}=0.022$ and $\mathrm{P}=0.038$, respectively). $\mathrm{RT}$-qPCR results for all six genes were consistent with the microarray data, therefore validating its reliability.

GKN1 (8.14-fold) was one of the most upregulated genes in body compared with groin skin in the present study (data not shown). The other two genes upregulated in body compared 
Table I. Primers used for qPCR validation.

Primer sequence (5'-3')

\begin{tabular}{|c|c|c|c|c|}
\hline Gene & Forward & Reverse & $\mathrm{Tm},{ }^{\circ} \mathrm{C}^{\mathrm{a}}$ & Target size, bp \\
\hline GAPDH $^{\mathrm{b}}$ & ACAGTCAAGGCAGAGAACGG & CCAGCATCACCCCACTTGAT & 60 & 98 \\
\hline AMP18 (GKN1) & TCAAGCCCTTGGTATGCTGG & TGAAGTCCGGCTTCTTGGTC & 60 & 198 \\
\hline CYP1A1 & TTTCACCCTCGCTCTGAAGG & AAGTTCTGTGGCCGAGATGG & 60 & 190 \\
\hline Connexin 43 & TTGTACCCGGGAGGAGACAT & CTGAGCCCCTCCAAAGACTG & 60 & 101 \\
\hline C-jun & GGATCAAGGCGGAGAGGAAG & CTGCGTTAGCATGAGTTGGC & 60 & 224 \\
\hline ITGB1 & AGCACGGATGAGGTGAACAG & ATCTCACAGGTTGGCCCTTG & 60 & 389 \\
\hline C3 & AACAAACGGGATCCCCTGAC & GAGTTCCCCTGCGTGTTGTA & 60 & 113 \\
\hline
\end{tabular}

${ }^{\mathrm{a}} \mathrm{Tm}$ represents the optimal temperature during PCR; ${ }^{\mathrm{h}} \mathrm{RNA}$ levels of GAPDH were assayed for normalization. qPCR, quantitative polymerase chain reaction; Tm, annealing temperature; AMP18, antrum muscle protein 18; GKN1, gastrokine 1; CYP1A1, cytochrome P450 family 1 subfamily A member 1; ITGB1, integrin subunit $\beta 1$; C3, complement component 3.

with groin skin, CYP1A1 (2.04-fold) and C-jun (2.04-fold), are involved in skin disease development and epidermal keratinocyte survival and differentiation $(24,25)$. Connexin 43 (-19.35-fold) and ITGB1 (-2.45-fold) are associated with differentiation of hair follicle stem cells $(26,27)$. The downregulated C3 gene (-8.92-fold) is a core member of the complement system (28).

Analysis of the region 1,800 bp upstream of the translation start site of DE genes. As presented in Fig. 3, three common motifs [41 bp (Fig. 3A), 50 bp (Fig. 3B) and 50 bp (Fig. 3C)] were identified in the upstream sequences of genes upregulated in body compared with groin skin. Notably, nuclear factor $1 \mathrm{~A}$ (NF-1A), Yi, E2 factor (E2F) and cyclic adenosine monophosphate response element binding (CREB)/CREB $\beta$ binding sites are present in the promoters of a number of upregulated genes. The promoter regions of genes downregulated in body compared with groin skin demonstrate 3 common motif sequences of 29 bp (Fig. 4A), 39 bp (Fig. 4B) and 50 bp (Fig. 4C). In these regions, binding sites were identified for the transcription factors LF-A1, Yi, E2F, Collier/Olfactory-1/early B-cell factor 1 (COE1) and peroxisome proliferator-activated receptor $\alpha(\operatorname{PPAR} \alpha)$. In addition, a binding site (the $\mathrm{U}$ site) was identified from the promoter regions of 12 downregulated genes, which has been previously demonstrated to be present in the olfactory cyclic nucleotide gated channel and type III cyclase promoters (29).

\section{Discussion}

In the present study, the molecular events associated with wool and cashmere growth control in the Laiwu black goat were investigated using microarray technology. Statistical analysis revealed hundreds of genes DE ( $>2$-fold-change) between the two sampled skin areas. RT-qPCR confirmed the reliability of the microarray results.

Heat shock protein 70.1 (Hsp70.1) is necessary and sufficient to accelerate depigmentation in vitiligo-prone Pmel-1 mice (30), while at least three different mutations of Agouti signaling peptide (ASIP) were hypothesized to cause the recessive black coat color pattern in sheep (31-33). A gene duplication affecting expression of the ovine ASIP gene is responsible for white and black sheep (32). In addition, Hsp70.1 was downregulated in body compared with groin skin of the Aohan fine-wool sheep (34). In the present study, Hsp70.1 in body skin may facilitate the regulation of the depigmentation of wool fiber. Upregulation of ASIP may have a similar role.

Overexpression of Hsp70.1 alone significantly inhibits aminoglycoside-induced hair cell death in mice (35). However, in the present study Hsp70.1 was downregulated in body skin of the Laiwu black goat. The reason for this contradiction remains to be elucidated.

Matrix metalloproteinase 2 (MMP2) activity is associated with the disappearance of collagen VII during the invasion of epithelial cords of hair follicles and sweat glands in human skin (36). MMP2 is involved in hair growth-associated extracellular matrix remodeling and cell migration, and may be a downstream effector, through which thymosin $\beta 4$ exerts its effect on hair growth (37). However, in the present study MMP2 was significantly downregulated in body compared with groin skin. A similar result was observed in the Aohan fine wool sheep (38), suggesting that MMP2 functions may differ in goat and sheep.

Integrins, including $\alpha 3, \alpha 11$ and $\beta 3$, have been demonstrated to be DE between primary and secondary hair follicles (11). In keratinocytes there was revealed to be a log-linear association between the relative expression levels of $\beta 1$ integrins (ITGB1) on the cell surface and proliferative capacity (39). In addition, ITGB1-mediated signaling is important in human hair growth control (40). Skin and hair follicle integrity is crucially dependent on ITGB1 expression in keratinocytes (41). However, ITGB1 expression in body skin of the Aohan fine wool sheep was downregulated $>10$ fold (38). The results of the present study were consistent with this, although the fold change was reduced at -2.45 . The underlying mechanism requires further investigation.

Rowe et al (24) identified the primary CYP1A1 location as the sebaceous gland surrounding the hair shaft. Consistent with this, in the present study CYP1A1 expression was upregulated in body skin according to the microarray and RT-qPCR data. 


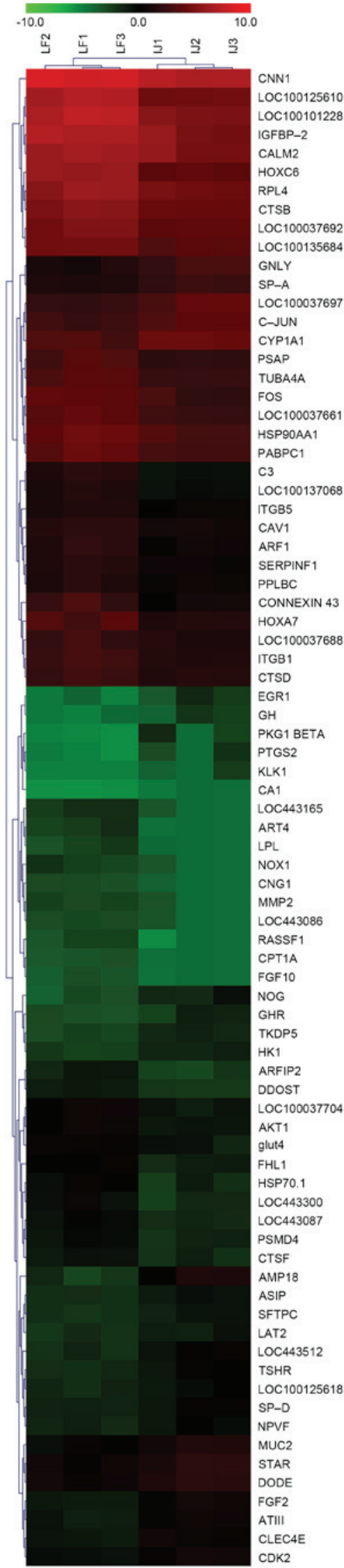

Figure 1. Hierarchical cluster analysis of genes differentially expressed in two skin areas of the Laiwu black goat. Each column represents one goat, and each row refers to a gene. Red indicates genes with a greater expression relative to the geometrical means and green indicates genes with a reduced expression relative to the geometrical means. LF1, LF2, LF3 represent the groin skin from three goats and IJ1, IJ2 and IJ3 represent the body skin from three goats.
Connexin 43 is a gap junction protein expressed in the dermal papilla (26). The G60S connexin 43 mutant regulates hair growth and hair fiber morphology in a mouse model of human oculodentodigital dysplasia (27). However, connexin 43 was downregulated in body skin in the microarray and RT-qPCR data of the present study. A similar pattern was observed in the Aohan fine wool sheep (38).

Fibroblast growth factor (FGF) 2 and -10 were upregulated and downregulated respectively in body compared with groin skin. FGF signaling is crucial for hair growth regulation $(42,43)$. FGF5, expressed in the outer root sheath, has been demonstrated to control anagen-catagen transition (44) via the inhibition of hair elongation (45). FGF21 and -22, which promote the transition of catagen (46), were reportedly downregulated in primary compared with secondary hair follicles of the Yunnan black goats (1). FGF expression varied throughout the hair follicle cycle, reflecting the role of FGFs in regulatory and developmental processes, which include patterning, morphogenesis, proliferation, differentiation and migration of cells (42). Expression analysis suggested an important role for FGF7 and -10, as well as their cognate receptor FGFR2-IIIb, in the proliferation and differentiation of the mature hair follicle (46).

Homeobox (Hox) A7 and HoxC6 were downregulated in body compared with groin skin in the present study. The first Hox gene demonstrated to have a universal role in hair follicle development was Hoxc13, as Hoxc13-deficient and -overexpressing mice exhibit severe hair growth and patterning defects (47). Members of the Hox family may perform essential and functionally diverse roles in hair that require complex transcriptional control mechanisms to ensure correct spatio-temporal patterns of Hox gene expression at homeostatic levels $(47,48)$. HOXA7 silences differentiation-specific genes during keratinocyte proliferation (49), which is consistent with the downregulation of HOXA7 in body skin compared with groin skin in the present study.

Growth hormone $(\mathrm{GH})$ and $\mathrm{GH}$ receptor $(\mathrm{GHR})$ were upregulated in body compared with groin skin, consistent with results in Aohan fine wool sheep (38). A number of growth factors have been demonstrated to be critical for hair growth, including FGFs, transforming growth factors, insulin-like growth factors (IGFs), epidermal growth factors and hepatocyte growth factor (50). The hair cycle in the dorsal skin of male GH-deficient rats enters a long-lasting telogen phase at 8 weeks of age; however, depilation induces a transient hair cycle (51). Therefore, GH and GHR may be involved in wool and cashmere growth regulation.

IGF2 is DE in anagen, catagen and telogen stages in the Shaanbei White cashmere goat (9). IGF binding protein (IGFBP) 5-mediated FGFR2-IIIb signaling is a critical regulator of the structure of the hair shaft medulla (46). The expression of IGFBP3 and IGFBP5 was decreased in early anagen and anagen phases (IGFBP3) or in catagen and telogen phases (IGFBP5) during a depilation-induced hair cycle (51). Downregulation of FGFR2-IIIb, an effect associated with increased expression levels of IGFBP5, was demonstrated to decrease the thickness of the hair shaft and to reduce the cellularity of the hair shaft medulla (46). In accordance with these results, IGFBP2 was downregulated in body compared with groin skin in the microarray data of the present study. 


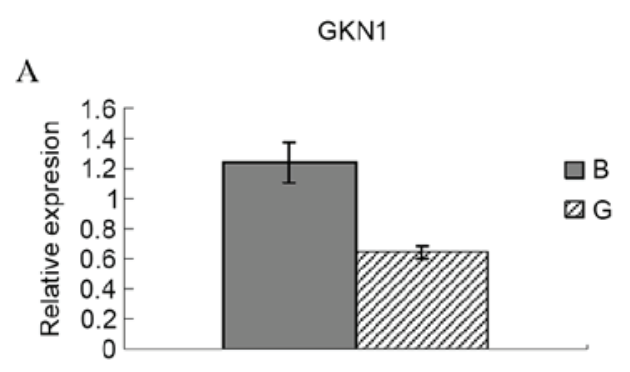

B

CYP1A1
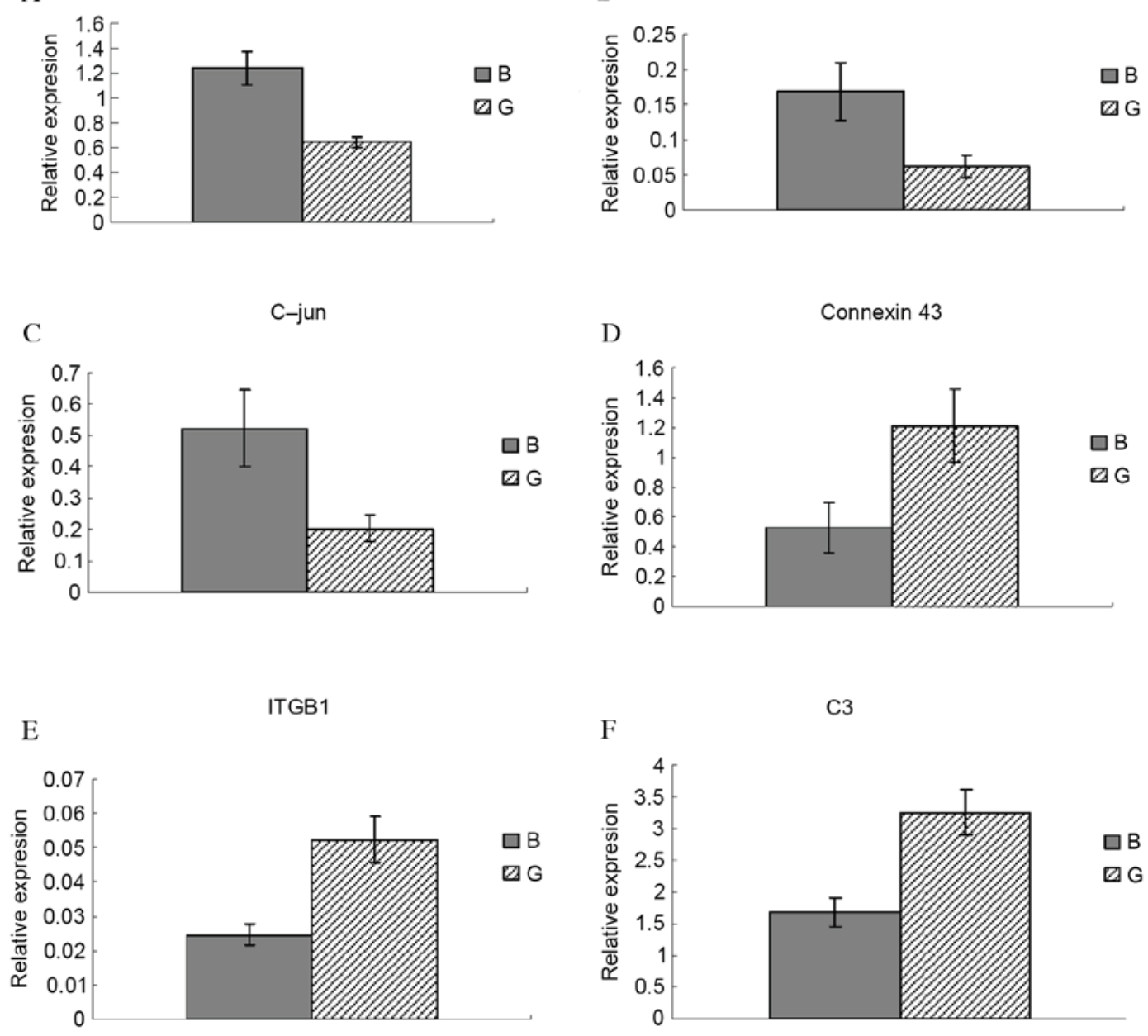

Figure 2. RT-qPCR verification of the microarray data. RT-qPCR was performed on skin samples to assess the relative expression of six differentially expressed genes. Student's $t$-tests were conducted to compare relative gene expression in body and groin skin. (A) GKN1 (P=0.013). (B) CYP1A1 (P=0.025). (C) $\mathrm{C}$-jun ( $\mathrm{P}=0.005)$. (D) Connexin 43 ( $\mathrm{P}=0.041)$. (E) ITGB1 ( $\mathrm{P}=0.022)$. (F) $\mathrm{C} 3$ ( $\mathrm{P}=0.038)$. RT-qPCR, reverse transcription polymerase chain reaction; GKN1, gastrokine 1; CYP1A1, cytochrome P450 family 1 subfamily A member 1 ; ITGB1, integrin subunit $\beta 1$; C3, complement component 3 ; B, body skin; G, groin skin.

In addition, the present study identified NF-1A, Yi, E2F and $\mathrm{CREB} / \mathrm{CREB} \beta$ binding sites in the promoter region of a number of upregulated genes. NF-1A negatively regulates target gene expression via binding to a silencer element (52); therefore, the reduced expression level of the gene subset may be caused by NF-1A upregulation or activation. CREB is a transcription factor important for keratinocyte proliferation (53-56). Adenosine stimulates growth of hair follicles by triggering phosphorylation of CREB (25). Combined recruitment of CREB, CCAAT-enhancer-binding protein $\beta$ and c-jun determines the activation of promoters upon keratinocyte differentiation (25). In the present study, no differential expression was observed in CREB between the two skin regions. Therefore, CREB may have important additional roles in wool and cashmere growth, potentially via phosphorylation or other modifications.

The promoter regions of downregulated genes demonstrated three common motif sequences of 29,39 and $50 \mathrm{bp}$. In these regions, binding sites were identified for the transcription factors LF-A1, Yi, E2F, COE1 and PPAR $\alpha$. The transcription factor LF-A1 is required for the cell-specific expression of the human $\alpha 1$-antitrypsin gene in hepatocytes (57). Binding sites of LF-A1 are present in the promoter regions of various genes expressed in the liver ( $\alpha 1$-antitrypsin, apolipoproteins $\mathrm{A} 1, \mathrm{~B} 1$ and A4, and pyruvate kinase). In the present study, thioredoxin-related transmembrane protein 2 , adiponectin, C1Q and collagen domain containing and perilipin 3 may associate with apolipoproteins. COE1 is essential for B-cell differentiation (58). Therefore, COE1 may be involved in the immune privilege process as described previously (14). Furthermore, PPAR $\alpha$ has been reported to contribute to hair growth and epidermal healing (59).

Notably, putative Yi and E2F sites were side-by-side in the upstream regions of upregulated and downregulated genes. $\mathrm{Yi}$ is an inducible DNA binding activity (60), consistent with its involvement in upregulated and downregulated genes. E2F has important roles in hair follicle growth, differentiation and survival $(61,62)$. E2F1-deficient mice have a high incidence of spontaneous epidermal tumors of hair follicle origin (63). 


\begin{tabular}{|c|c|c|c|c|}
\hline Name & Strand & Start & P-value & Sites \\
\hline CYBB (LOC102175938)- & + & 728 & $1.77 \mathrm{e}-25$ & GGATTCTCCA GGCAAGAATACTGGAGTGGGTTGCCATTTCCTTCTCCAGGG GAATCTTCCT \\
\hline TSHR & + & 1593 & $1.02 \mathrm{e}-22$ & GAGTTCTCCA GGCAAGAATATTGGAGTGGGTTGCCATTCCCTTCTCCAGAG GAACTTCCTA \\
\hline ITGA4 & + & 1140 & $7.17 \mathrm{e}-22$ & AGATTTTCCA GGCAAGAATACTGGAGTGGGTTGCCATGCCCTCTTCCAGAG GATCTTCTGA \\
\hline CLEC4E- & - & 1110 & $2.12 \mathrm{e}-21$ & GAATTTTCCA AGCTAGAATACTGGAGTGGGTTGCCGTTTCCTTCTCCAGGG AATCTTCCCG \\
\hline ASIP & + & 1034 & $2.35 \mathrm{e}-21$ & GGATTCTCCA GGCAAGAATACTGGGGTGGGTTGCCATGCCCTCCTTCAGGG GATCTTCCCA \\
\hline GKN1 & + & 1193 & $1.35 \mathrm{e}-20$ & GGATTTCCCA GGCAAGAATACTGGAGTGGGTTACTATTTCCACCTCCAGAG GGTCTTTCTG \\
\hline ABCF1- & + & 679 & $2.03 e-20$ & GAATTTTCCA GGCAAGAGTACCGGAGTGGGTTGCCATTTCCTTCTCCATGA AGTCTTTAAA \\
\hline EPRS- & + & 398 & $7.91 \mathrm{e}-19$ & GGATTTTCCA AGCAAGAATACCGGAGCGGATTGCCATTTTCTTCTCCAGCG GATCTTCCTG \\
\hline SFTPA1 (LOC102175746)- & - & 394 & $1.27 e-14$ & TATGAGGGAC AGACAGAATGAGTGAGTGAGTGACCATTTCCTTCCTCCCAG CAACCTGGGA \\
\hline GH- & + & 168 & $1.07 e-13$ & TTCCCTCCAG GGACCTAAGCCAGGAGAGGGTGGGCATGGCCCCCAGCATGG AAGGGACCTA \\
\hline
\end{tabular}

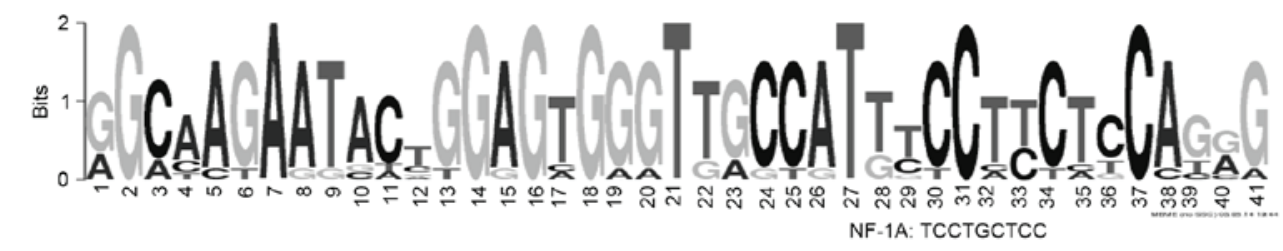

\begin{tabular}{|c|c|c|c|c|}
\hline Name & Strand & Start & P-value & Sites \\
\hline YBB (LOC & + & 655 & $.63 e-27$ & GC TCCTCCGTCC \\
\hline KN1 & + & 1120 & $5.96 \mathrm{e}-25$ & CGCCCATTTG CTCAGTTGTGACCAACTCTTTGTAACCCCGTGGACTGTAGCCCACCAGGC TCCTCCATCC \\
\hline EC4E- & + & 526 & $.70 \mathrm{e}-23$ & GGTTCAGTTG C \\
\hline BCF1. & + & 1068 & $8.40 \mathrm{e}-23$ & GTTCAGTCA CTCAGTTGTGTCCAACTCTTTGAGACCTCACAAACTGCAGCACTCAAG \\
\hline SIP & + & 961 & e-23 & ITCC \\
\hline KDP5 & + & 453 & $2.72 \mathrm{e}-22$ & TGTTCAGTCA \\
\hline PRS- & + & 324 & $3.70 \mathrm{e}-22$ & AGTGAAGTCG CTCTGTCGTGTCTGACTCGT \\
\hline SF2 (LOC102186892)- & + & 761 & $12 \mathrm{e}-20$ & GTTCAGCCT TTAAGTCCTATCTGACTCTTTGCAACATCATGGACTGCAGCACGCTAGGC TTCCCTGTCC \\
\hline HRR & + & 1520 & $82 \mathrm{e}-15$ & AGTCCAAC TCTGAGCGACTCTTTGCATCTGCGATCCCACAGACTATAGCCCACCAGAC TTCTCTGTCC \\
\hline K1 & + & 144 & $.17 \mathrm{e}-14$ & СCCTGCTTC CTGGGGCCTGGACAACCTTCTCCCACCCCAAGAGCTGTGGAACAGACGGG ACACTGAGCA \\
\hline
\end{tabular}

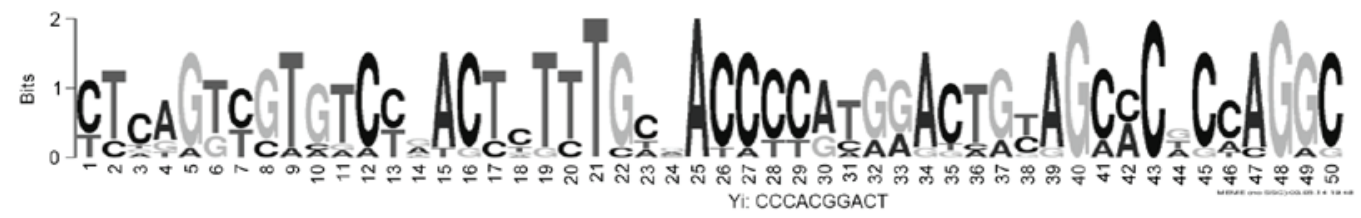

E2F: CCCGCCAGG

$\begin{array}{lcccc}\text { C Name } & \text { Strand } & \text { Start } & \text { P-value } & \text { Sites } \\ \text { CYBB (LOC102175938) } & + & 773 & 2.16 \mathrm{e}-26 & \text { CCAGGGGAAT CTTCCTGACCCAGAGATCAAACCCAGGTCTCCCACATTGCAGGCAGACGC TTTAACCTCT } \\ \text { EPRS- } & + & 442 & 6.55 \mathrm{e}-24 & \text { TCCAGCGGAT CTTCCTGACCTAGGGATCGAACTCAGGTCTCCCGCATTGCGGACAGACTC TACCGTCTAA } \\ \text { CLEC4E- } & - & 1057 & 2.27 \mathrm{e}-23 & \text { TCCAGGGAAT CTTCCCGAACCAGGAATCGAACCCAGGTCTCCCTCACTAAAGGCAGACTC TTTACCAGGT } \\ \text { SERPINC1- } & + & 472 & 4.11 \mathrm{e}-23 & \text { TCCAGGGGAT CTTCCTGACCCAGAAATTGAACCTAGGTCTCTGGCATTGGAGGCAGATTC TTTACCATCT } \\ \text { ITGA4 } & + & 1183 & 4.53 \mathrm{e}-23 & \text { TTCCAGAGGA TCTTCTGACTCAGGGATCAAACCAAGGTCTCCTGCATTGCAGGCAGATTC ACAAAGTTTC } \\ \text { TSHR } & + & 1637 & 7.97 \mathrm{e}-23 & \text { TCCAGAGGAA CTTCCTAACCCAGGGATCGAACCCTTGTCTCCTATGTCACAGGCAGATTC TTTACCGCTT } \\ \text { ASIP } & - & 38 & 1.27 \mathrm{e}-19 & \text { CATGTAGAAT CTTCCTGGACCAGGGATCAAACCCATGTCCCCTGCATTGGCAGGTGGTT CTTATCCACT } \\ \text { GKN1 } & + & 1237 & 1.75 \mathrm{e}-17 & \text { TCCAGAGGGT CTITCTGACCCAAGAATCCAACCCACCTTTCCTTTGTCTGCTGCACTGAC AGATTCTCTA }\end{array}$

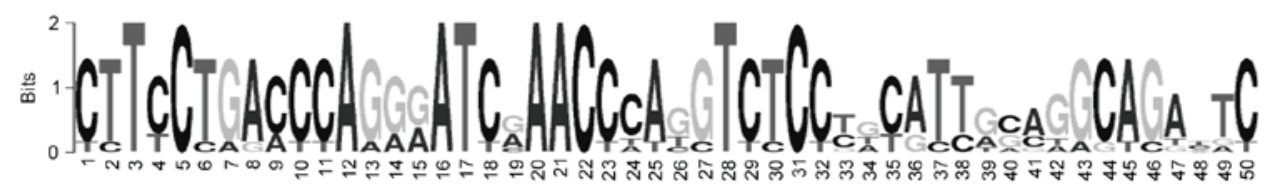

CREB CREBbeta: CTGACGCAG

Figure 3. Demonstrates 3 common motifs in 1,800 base pairs upstream of the translation start site in genes upregulated in body compared with groin skin. Strands + and - denote the sense and antisense DNA sequence. Start denotes the number of base pairs upstream of the transcriptional start site. P-value denotes the probability of the motif occurring by random chance. At the bottom of each panel the cis-regulatory elements for various transcription factors are presented, which may bind to the common motif of the genes listed. Common motifs containing (A) NF-1A, (B) Yi and E2F, and (C) CREB/CREB $\beta$ are represented. NF-1A, nuclear factor 1A; E2F, E2 factor; CREB, cyclic adenosine monophosphate response element binding.

However, the reason for the existence of putative E2F sites in upregulated and downregulated genes requires further investigation. The $\mathrm{U}$ site was present in the promoter regions of 12 downregulated genes, of which 11 contained COE1 binding sites, consistent with a previous study (29).
A previous study in sheep revealed that the number and fold-change of DE genes in December were markedly reduced compared with August (21), which is consistent with the decreased activity of hair follicles in winter. Di et al (64) investigated DE genes of skin tissue in fine wool sheep with 


\begin{tabular}{|c|c|c|c|c|}
\hline $\begin{array}{l}\text { Name } \\
\text { LOC102184629 }\end{array}$ & Strand & $\begin{array}{l}\text { Start } \\
19\end{array}$ & $\begin{array}{l}\text { P-value } \\
1.90 \mathrm{e}-17\end{array}$ & $\begin{array}{l}\text { Sites } \\
\text { ATGGACAGAG GAGCCTGGCGGGTACAGTTCATGGGGTC ACATAGAGTC }\end{array}$ \\
\hline TRPV6- & + & 1052 & $2.80 \mathrm{e}-17$ & ATGGGCAGAG GAGCCTGGCGGGCTACAGTCCATGGGGTG GCAAAGAGCT \\
\hline PSAP- & + & 800 & $7.70 \mathrm{e}-17$ & ATGGACAGAG GAGCCTGGCGGGCTGCAGTCCATGGGGTG GCAAAGAGTC \\
\hline GJA1 (co & + & 627 & $1.95 \mathrm{e}-16$ & ATGGACAGAG AAGCCTGGCCGGCTACAGTCCATGGGGTC ACAAAGAGTC \\
\hline TMX2 & - & 776 & 15 & AA GA \\
\hline GUCY & - & 357 & $43 e-15$ & ATGGACAGAG GAGCCTGGCAGGCTACAGTGCATAGGGTC ACAA \\
\hline CA3- & 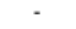 & 471 & $6.27 \mathrm{e}-15$ & AGGGATATGG AAGCCTGGCGTGCCGCAGTCCATGGGGTC AT/ \\
\hline ADIPOQ & - & 331 & $6.27 e-15$ & ACAGACAGA GAGCCTTGTGGGCTACAGTTCATAGGGTC GCGAAGAGTA \\
\hline HSPA1L & - & 888 & $6.91 \mathrm{e}-15$ & AGGGACAGCG GAGCCTGGCGGGCTGCCGTCTATGGGGTC GCAC \\
\hline PLIN3 & t & 1428 & $8.95 e-15$ & ACGGACAGAG GAGCCTGGTGGGATACAGTCCACAGGGTC ACAAAC \\
\hline DGKZ & ${ }^{+}$ & 684 & $1.06 \mathrm{e}-14$ & AAGGACAGGG AAGCCTGGTGTGCTGGAGTCCATGGGGTC GCA \\
\hline NOMO1 & - & 121 & $1.93 \mathrm{e}-14$ & ATGGACAGAG GAGCCTGATGGGCTACAATCCATGGGGTT GCAATAGTCA \\
\hline LOC102175922 & - & 1250 & $2.46 \mathrm{e}-14$ & ATGGACAGAG GAGCCTGGTGGGCTATAGTCCACAGGGTT GCAAAGATTC \\
\hline D19 & $\cdot$ & 952 & $4.30 \mathrm{e}-14$ & CGGTT ACAAAGACTC \\
\hline $\mathrm{ZC} 3 \mathrm{H} 11$ & - & 45 & $2.34 \mathrm{e}-12$ & ATGGACAGGG AGACCTGGCATGCTGCAGTCCATGGGCTC GCAGAGTCAG \\
\hline C1- & - & 1016 & $4 e-11$ & AAAGAGTGG \\
\hline
\end{tabular}

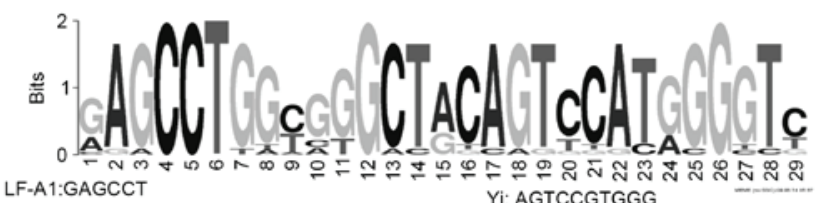

E2F: CCT GGCGGG

\begin{tabular}{|c|c|c|c|c|c|c|}
\hline Name & Strand & Start & P-value & & Sites & \\
\hline NOMO1 & - & 179 & $2.15 \mathrm{e}-23$ & TTGGGTCAGA & AAGATCCCCTGGAGAAGGAAATGGCAACCCACTCCAGTA & TTCTTGCCAG \\
\hline LOC102175922 & + & 961 & $6.55 \mathrm{e}-23$ & CTGGGTCAGG & AAGGTCCCCTGGAGAAGGGAATGGCTACCCACTCCAGTA & TTCTTGCCTG \\
\hline PNRC1- & - & 1047 & $.55 e-23$ & TTGGGTTCGG & AAGATTCCCTGGAGAAGGGAATGGCAACCCACTCCAGTA & TGCTTGCCTG \\
\hline PLIN3 & . & 63 & $1.55 \mathrm{e}-21$ & CTGGGTCGGG & AAGATCCCCTGGAGAAGGAAATGGCAGCCCACTCCAGTA & TTCCTGCCTG \\
\hline TRPV6- & + & 984 & $1.13 e-20$ & CTGTGCTGGG & AAGAATCCGCTGGAGAAGGGAATGGCTACCCACTCCAGTC & СTCTTGCCTG \\
\hline GUCY1B3. & - & 1127 & $1.06 \mathrm{e}-19$ & CTGGGTTGGG & AAGATCCCCTGGAGAAGGGAAAGGTTACCCACTCTAGTA & TTCTGGCCTA \\
\hline SH3D 19 & - & 1010 & $2.23 \mathrm{e}-19$ & CTGGGTTGGA & AAGATTCCCTGGAGTAGGAAATGGCTACCCACTGCAGTA & TTCTTGGCTG \\
\hline LOC1021 & - & 78 & $5.18 \mathrm{e}-19$ & CTGGGTCGGG & AAGATCCCTTGGAGGAGTGCATGGCAACCCACTCCATTA & TTCTTGCCTG \\
\hline PSAP. & + & 732 & $4,69 \mathrm{e}-18$ & TATTGGGTGG & AAGATCCCCTGGAGAAGGGCATGACAAGTGACTCCAGTA & TTCTGGCCTG \\
\hline GJA1 (connexin) & + & 559 & $05 \mathrm{e}-17$ & CTGAGTTGAG & AGGATCCCATGGAAAAGAGAATGGCTATTCACTCCAGTA & TTCTTACCTG \\
\hline ADIPOQ & - & 388 & $6.86 \mathrm{e}-17$ & CTGGGTCAGG & AAGATCTCCCGGAGGAGGGCATGGCAATTCACCCCATTA & TTCTTGCCAG \\
\hline TMX2 & - & 834 & $1.47 \mathrm{e}-16$ & CCTCAGGTGA & GAAGAGCCCTGGAGAAGGGAAAGGCTACCCACTGCATTA & TCССTGCTTG \\
\hline DGKZ & & 309 & $1.64 \mathrm{e}-12$ & GACCAAACGT & GGTCCCCTGGGGAAGGGGGAATGTGCAAACCACTTCAGTA & TTCTTGCCTT \\
\hline SPA1L & & 946 & $6.69 \mathrm{e}-12$ & SCTCTATTAG & GATTGTTTCAGAGAAAGCAATGGTACCCCACTCCAATG & CTCTTGCCTG \\
\hline
\end{tabular}
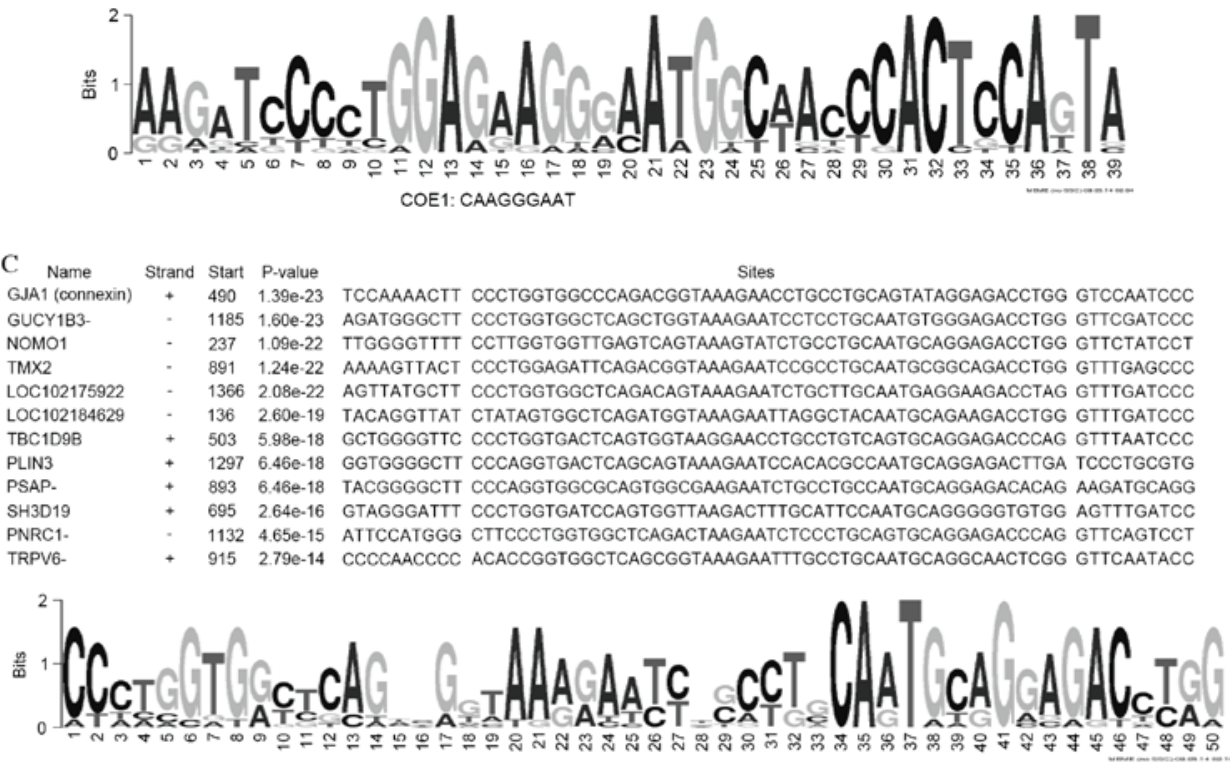

RATSAC3_02 \& RATSOCNC_02: CTGCCAGCA

PPAR-alpha: AGACCT

Figure 4. Demonstrates 3 common motifs in 1,800 base pairs upstream of the translation start site in genes downregulated in body compared with groin skin. Strands + and - denote the sense and antisense DNA sequence. Start denotes the number of base pairs upstream of the transcriptional start site. P-value denotes the probability of the motif occurring by random chance. At the bottom of each panel the cis-regulatory elements for various transcription factors are presented, which may bind to the common motif of the genes listed. Common motifs containing (A) LF-A1, E2F and Yi, (B) COE1, and (C) PPAR $\alpha$ are represented. E2F, E2 factor; COE1, Collier/Olfactory-1/early B-cell factor 1; PPAR $\alpha$, peroxisome proliferator-activated receptor $\alpha$.

various fiber diameters using the Agilent sheep gene expression microarray. Therefore, the present study validates the use of Agilent Sheep Gene Expression Microarray in wool follicle investigations and provides a strong rationale for future studies. 
Brenaut et al (16) investigated the contribution of mammary epithelial cells to the early stages of an immune response against Staphylococcus aureus in goats using the Agilent sheep gene expression microarray. Goyal et al (65) conducted microarray with advanced bioinformatic analysis on carotid arteries from the normoxic near-term ovine fetus at sea level and high altitude. These studies identified genes and networks involved in the investigated processes. Therefore, microarray technology may be used to examine various aspects of the sheep and goat species.

The present study used microarray methodologies to investigate hair and cashmere growth in the Laiwu black goat. Gene transcripts expressed in skin cells from hair-rich and hairless areas were compared, and hundreds of DE genes were identified. However, no keratin or keratin-associated protein genes were DE, which contradicts the results of a previous study (34). Whether this problem is the result of species differences in probe sets remains to be elucidated.

Gene annotation is incomplete and imperfect (certain DE genes remain to be identified), which may explain why certain factors, including keratin and keratin-associated proteins, were not identified in the present study. However, the repertoire of gene probes used $(8 \mathrm{x} 15 \mathrm{~K})$ confirmed the involvement of a number of growth factors and HOX genes. The application of an original algorithm to construct gene networks of temporal regulation of hair growth was not performed due to limited DE genes. The present study identified molecules in the cashmere-bearing skin area of the Laiwu black goat, which may contribute to hair and cashmere traits.

\section{Acknowledgements}

The present study was funded by the National Hair Sheep Industry Technology System (grant no. CARS-40), Projects of Qingdao People's Livelihood Science and Technology (grant nos. 13-1-3-88-nsh and 14-2-3-45-nsh) and the National Natural Science Foundation of China (grant no. 31301936).

\section{References}

1. Dong Y, Xie M, Jiang Y, Xiao N, Du X, Zhang W, Tosser-Klopp G, Wang J, Yang S, Liang J, et al: Sequencing and automated whole-genome optical mapping of the genome of a domestic goat (Capra hircus). Nat Biotechnol 31: 135-141, 2013.

2. Horner ME, Parkinson KE, Kaye V and Lynch PJ: Dowling-Degos disease involving the vulva and back: Case report and review of the literature. Dermatol Online J 17: 1, 2011.

3. Lueking A, Huber O, Wirths C, Schulte K, Stieler KM, Blume-Peytavi U, Kowald A, Hensel-Wiegel K, Tauber R, Lehrach $\mathrm{H}$, et al: Profiling of alopecia areata autoantigens based on protein microarray technology. Mol Cell Proteomics 4: 1382-1390, 2005.

4. Purvis IW and Franklin IR: Major genes and QTL influencing wool production and quality: A review. Genet Sel Evol 37 (Suppl 1): S97-S107, 2005.

5. Cano EM, Marrube G, Roldan DL, Bidinost F, Abad M, Allain D, Vaiman D, Taddeo H and Poli MA: QTL affecting fleece traits in Angora goats. Small Ruminant Research 71: 158-164, 2007.

6. Adams $\mathrm{N}$ and Cronjé $\mathrm{P}$ : A review of the biology linking fibre diameter with fleece weight, liveweight, and reproduction in Merino sheep. Australian Journal of Agricultural Research 54: $1-10,2003$

7. Wenguang Z, Jianghong W, Jinquan L and Yashizawa M: A subset of skin-expressed microRNAs with possible roles in goat and sheep hair growth based on expression profiling of mammalian microRNAs. OMICS 11: 385-396, 2007.
8. Rufaut NW, Pearson AJ, Nixon AJ, Wheeler TT and Wilkins RJ: Identification of differentially expressed genes during a wool follicle growth cycle induced by prolactin. J Invest Dermatol 113: 865-872, 1999 .

9. Wang HR, Feng ZC, Du M, Ren JK and Li HR: Initial research for seasonal variation of wool growth of Aohan fine wool sheep. Inner Mong Anim Sci 3: 1-3, 1994 (In Chinese).

10. McElwee KJ and Sinclair R: Hair Physiology and its disorders. Drug discovery today: Disease Mechanisms 5: e163-e171, 2008.

11. Zhu B, Xu T, Yuan J, Guo X and Liu D: Transcriptome sequencing reveals differences between primary and secondary hair follicle-derived dermal papilla cells of the Cashmere goat (Capra hircus). PLoS One 8: e76282, 2013.

12. Zhu B, Xu T, Zhang Z, Ta N, Gao X, Hui L, Guo X and Liu D: Transcriptome sequencing reveals differences between anagen and telogen secondary hair follicle-derived dermal papilla cells of the Cashmere goat (Capra hircus). Physiol Genomics 46: 104-111, 2014.

13. Wu Z, Fu Y, Cao J, Yu M, Tang X and Zhao S: Identification of differentially expressed miRNAs between white and black hair follicles by RNA-sequencing in the goat (Capra hircus). Int J Mol Sci 15: 9531-9545, 2014.

14. Liu N, Li H, Liu K, Yu J, Cheng M, De W, Liu J, Shi S, He Y and Zhao J: Differential expression of genes and proteins associated with wool follicle cycling. Mol Biol Rep 41: 5343-5349, 2014.

15. Menzies M, Stockwell S, Brownlee A, Cam G and Ingham A: Gene expression profiles of BMP4, FGF10 and cognate inhibitors, in the skin of foetal Merino sheep, at the time of secondary follicle branching. Exp Dermatol 18: 877-879, 2009.

16. Brenaut P, Lefèvre L, Rau A, Laloë D, Pisoni G, Moroni P, Bevilacqua $\mathrm{C}$ and Martin P: Contribution of mammary epithelial cells to the immune response during early stages of a bacterial infection to Staphylococcus aureus. Vet Res 45: 16, 2014.

17. Geng R, Yuan C and Chen Y: Exploring differentially expressed genes by RNA-Seq in cashmere goat (Capra hircus) skin during hair follicle development and cycling. PLoS One 8: e62704, 2103.

18. Nacht M, Dracheva T, Gao Y, Fujii T, Chen Y, Player A, Akmaev V, Cook B, Dufault M, Zhang M, et al: Molecular characteristics of non-small cell lung cancer. Proc Natl Acad Sci USA 98: 15203-15208, 2001.

19. Tang Z, Li Y, Wan P, Li X, Zhao S, Liu B, Fan B, Zhu M, Yu M and Li K: LongSAGE analysis of skeletal muscle at three prenatal stages in Tongcheng and Landrace pigs. Genome Biol 8: R115, 2007.

20. Dennis G Jr, Sherman BT, Hosack DA, Yang J, Gao W, Lane HC and Lempicki RA: DAVID: Database for annotation, visualization, and integrated discovery. Genome Biol 4: P3, 2003.

21. Liu N, Li H, Liu K, Yu J, Bu R, Cheng M, De W, Liu J, He G and Zhao J: Identification of skin-expressed genes possibly associated with wool growth regulation of Aohan fine wool sheep. BMC Genet 15: 144, 2014.

22. Bailey TL, Boden M, Buske FA, Frith M, Grant CE, Clementi L, Ren J, Li WW and Noble WS: MEME SUITE: Tools for motif discovery and searching. Nucleic Acids Res 37: W202-W208, 2009.

23. Matys V, Kel-Margoulis OV, Fricke E, Liebich I, Land S, Barre-Dirrie A, Reuter I, Chekmenev D, Krull M, Hornischer K, et al: TRANSFAC and its module TRANSCompel: Transcriptional gene regulation in eukaryotes. Nucleic Acids Res 34 (Database Issue): D108-D110, 2006.

24. Rowe JM, Welsh C, Pena RN, Wolf CR, Brown K and Whitelaw $\mathrm{CB}$ : Illuminating role of CYP1A1 in skin function. J Invest Dermatol 128: 1866-1868, 2008.

25. Maimaiti A: Genetic polymorphism of five KAP gene and their associations with wool quality Traitsin Chinese merino sheep (Xinjiang type) group. PhD dissertation, Xinjiang Agricultural University. Globe Thesis, 2013.

26. Higgins CA, Richardson GD, Ferdinando D, Westgate GE and Jahoda CA: Modelling the hair follicle dermal papilla using spheroid cell cultures. Exp Dermatol 19: 546-548, 2010.

27. Churko JM, Chan J, Shao Q and Laird DW: The G60S connexin 43 mutant regulates hair growth and hair fiber morphology in a mouse model of human oculodentodigital dysplasia. J Invest Dermatol 131: 2197-2204, 2011.

28. Alcorlo M, López-Perrote A, Delgado S, Yébenes H, Subías M, Rodríguez-Gallego C, Rodríguez de Córdoba S and Llorca O: Structural insights on complement activation. FEBS J 282: 3883-3891, 2015.

29. Chang $\mathrm{CH}$, Jiang TX, Lin CM, Burrus LW, Chuong CM and Widelitz R: Distinct Wnt members regulate the hierarchical morphogenesis of skin regions (spinal tract) and individual feathers. Mech Dev 121: 157-171, 2004. 
30. Mosenson JA,Zloza A,Klarquist J, Barfuss AJ, Guevara-Patino JA and Poole IC: HSP70i is a critical component of the immune response leading to vitiligo. Pigment Cell Melanoma Res 25: $88-98,2012$

31. Peñagaricano F, Zorrilla P, Naya H, Robello C and Urioste JI: Gene expression analysis identifies new candidate genes associated with the development of black skin spots in Corriedale sheep. J Appl Genet 53: 99-106, 2012.

32. Norris BJ and Whan VA: A gene duplication affecting expression of the ovine ASIP gene is responsible for white and black sheep. Genome Res 18: 1282-1293, 2008.

33. Hammond NL, Headon DJ and Dixon MJ: The cell cycle regulator protein $14-3-3 \sigma$ is essential for hair follicle integrity and epidermal homeostasis. J Invest Dermatol 132: 1543-1553, 2012.

34. Zhao J, Li H, Liu K, Liu K, Liu Zuo and Li J: Differential expression of immune genes between body side skin and groin skin of Aohan fine wool sheep. Agric Sci Technol 12: 2475-2479, 2012.

35. Taleb M, Brandon CS, Lee FS, Lomax MI, Dillmann WH and Cunningham LL: Hsp70 inhibits aminoglycoside-induced hair cell death and is necessary for the protective effect of heat shock. J Assoc Res Otolaryngol 9: 277-289, 2008.

36. Karelina TV, Bannikov GA and Eisen AZ: Basement membrane zone remodeling during appendageal development in human fetal skin. The absence of type VII collagen is associated with gelatinase-A (MMP2) activity. J Invest Dermatol 114: 371-375, 2000

37. Philp D, Nguyen M, Scheremeta B, St-Surin S, Villa AM, Orgel A, Kleinman HK and Elkin M: Thymosin beta4 increases hair growth by activation of hair follicle stem cells. FASEB J 18 : 385-387, 2004

38. Zhao J, Liu N, Liu K, He J, Yu J, Bu R, Cheng M, De W, Liu J and $\mathrm{Li} \mathrm{H}$ : Identification of genes and proteins associated with anagen wool growth. Animal Genetics (In Press).

39. Jones PH and Watt FM: Separation of human epidermal stem cells from transit amplifying cells on the basis of differences in integrin function and expression. Cell 73: 713-724, 1993.

40. Kloepper JE, Hendrix S, Bodó E, Tiede S, Humphries MJ, Philpott MP, Fässler R and Paus R: Functional role of beta 1 integrin-mediated signalling in the human hair follicle. Exp Cell Res 314: 498-508, 2008

41. Brakebusch C, Grose R, Quondamatteo F, Ramirez A, Jorcano JL, Pirro A, Svensson M, Herken R, Sasaki T, Timpl R, et al: Skin and hair follicle integrity is crucially dependent on beta 1 integrin expression on keratinocytes. EMBO J 19: 3990-4003, 2000.

42. Galbraith $\mathrm{H}$ : Fundamental hair follicle biology and fine fibre production in animals. Animal 4: 1490-1509, 2010.

43. Rosenquist TA and Martin GR: Fibroblast growth factor signalling in the hair growth cycle: Expression of the fibroblast growth factor receptor and ligand genes in the murine hair follicle. Dev Dyn 205: 379-386, 1996.

44. Botchkarev VA and Paus R: Molecular biology of hair morphogenesis: Development and cycling. J Exp Zool B Mol Dev Evol 298: 164-180, 2003.

45. Hebert JM, Rosenquist T, Götz J and Martin GR: FGF5 as a regulator of the hair growth cycle: Evidence from targeted and spontaneous mutations. Cell 78: 1017-1025, 1994.

46. Schlake T: FGF signals specifically regulate the structure of hair shaft medulla via IGF-binding protein 5. Development 132: 2981-2990, 2005.

47. Awgulewitsch A: Hox in hair growth and development. Naturwissenschaften 90: 193-211, 2003.

48. Stelnicki EJ, Kömüves LG, Kwong AO, Holmes D, Klein P, Rozenfeld S, Lawrence HJ, Adzick NS, Harrison M and Largman C: HOX homeobox genes exhibit spatial and temporal changes in expression during human skin development. J Invest Dermatol 110: 110-115, 1998
49. La Celle PT and Polakowska RR: Human homeobox HOXA7 regulates keratinocyte transglutaminase type 1 and inhibits differentiation. J Biol Chem 276: 32844-32853, 2001.

50. Stenn KS and Paus R: Controls of hair follicle cycling. Physiol Rev 81: 449-494, 2001.

51. Umeda-Ikawa A, Shimokawa I and Doi K: Time-course expression profiles of hair cycle-associated genes in male mini rats after depilation of telogen-phase hairs. Int J Mol Sci 10: 1967-1977, 2009

52. Lee JS, Xiao J, Patel P, Schade J, Wang J, Deneen B, Erdreich-Epstein A and Song HR: A novel tumor-promoting role for nuclear factor IA in glioblastomas is mediated through negative regulation of p53, p21, and PAI1. Neuro Oncol 16: 191-203, 2014.

53. Jang SI and Steinert PM: Loricrin expression in cultured human keratinocytes is controlled by a complex interplay between transcription factors of the Sp1, CREB, AP1, and AP2 families. J Biol Chem 277: 42268-42279, 2002.

54. Sander GR and Powell BC: Structure and expression of the ovine Hoxc-13 gene. Gene 327: 107-116, 2004.

55. Tanaka S, Miura I, Yoshiki A, Kato Y, Yokoyama H, Shinogi A, Masuya H, Wakana S, Tamura M and Shiroishi T: Mutations in the helix termination motif of mouse type I IRS keratin genes impair the assembly of keratin intermediate filament. Genomics 90: 703-711, 2007.

56. Ansari KM, Rundhaug JE and Fischer SM: Multiple signaling pathways are responsible for prostaglandin E2-induced murine keratinocyte proliferation. Mol Cancer Res 6: 1003-1016, 2008.

57. Langbein L, Rogers MA, Praetzel-Wunder S, Helmke B, Schirmacher P and Schweizer J: K25 (K25irs1), K26 (K25irs2), K27 (K25irs3), and K28 (K25irs4) represent the type I inner root sheath keratins of the human hair follicle. J Invest Dermatol 126: 2377-2386, 2006

58. Lin H and Grosschedl R: Failure of B-cell differentiation in mice lacking the transcription factor EBF. Nature 376: 263-267, 1995.

59. Liu G, Liu R, Li Q, Tang X, Yu M, Li X, Cao J and Zhao S: Identification of microRNAs in wool follicles during anagen, catagen, and telogen phases in Tibetan sheep. PLoS One 8: e77801, 2013

60. Jiang Y, Xie M, Chen W, Talbot R, Maddox JF, Faraut T, Wu C, Muzny DM, Li Y, Zhang W, et al: The sheep genome illuminates biology of the rumen and lipid metabolism. Science 344: 1168-1173, 2014

61. Yu ZD, Bawden CS, Henderson HV, Nixon AJ, Gordon SW and Pearson AJ: Micro-arrays as a discovery tool for wool genomics. Proceedings of the New Zealand Society of Animal Production 66: 129-133, 2006.

62. Xu T, Guo X, Wang H, Du X, Gao X and Liu D: De novo transcriptome assembly and differential gene expression profiling of three Capra hircus skin types during Anagen of the hair growth cycle. Int J Genomics 2013: 269191, 2013.

63. Yu ZD, Gordon SW, Pearson AJ, Henderson HV, Craven AJ and Nixon AJ: Gene expression profiling of wool follicle growth cycles by cDNA microarray. Proceedings of the New Zealand Society of Animal Production 68: 39-42, 2008.

64. Di J, La Z, Xu X, Zhang Y, Tian K, Tian Y, Yu L and Ha N: Genome array on differentially expressed genes of skin tissue in fine wool sheep with different fiber diameter. Acta Veterinaria Et Zootechnica Sinica 5: 681-689, 2013 (In Chinese).

65. Goyal R, Van Wickle J, Goyal D, Matei N and Longo LD: Antenatal maternal long-term hypoxia: Acclimatization responses with altered gene expression in ovine fetal carotid arteries. PLoS One 8: e82200, 2013. 